Artigo Original

\title{
Diagnóstico de síndromes parkinsonianas em uma clínica brasileira de distúrbios do movimento
}

\section{Diagnostic of parkinsonian syndrome in a Brazilian movement disorders clinic}

\author{
Elton Gomes Silva ${ }^{1}$, Maura Aparecida Viana², Elizabeth Maria Aparecida Barasnevicius \\ Quagliato $^{3}$
}

\section{RESUMO}

Introdução: Nosso trabalho visou observar a freqüência das etiologias na população de pacientes com síndrome parkinsoniana (SP). Casuística e método: Foram analisados retrospectivamente prontuários de pacientes com SP atendidos no Hospital das Clínicas da Universidade Estadual de Campinas entre Janeiro de 1999 e Dezembro de 2001, avaliando-se quadro clínico e exames neuroimagem. Resultados: Dos 177 prontuários analisados, 151 (85,3\%) apresentavam diagnóstico de doença de Parkinson (DP), 6 (3,4\%) parkinsonismo vascular (PV), 7 (4,0\%) parkinsonismo induzido por drogas (PID), 6 (3,4\%) Parkinson-plus (PP), 1 (0,6\%) deficiência de pantetonato-quinase, 1 (0,6\%) ataxia espinocerebelar forma 3 , e 5 (2,8\%) SP indeterminada (PI). A idade de início da doença foi de $54 \pm 12$ anos na DP, $75 \pm 9$ no PID, $60 \pm 14$ no PV e $53 \pm 9$ no PP. Conclusão: A DP foi a forma de SP mais comum em nosso ambulatório, seguido por PID, PV e PP. Os pacientes com PID tiveram idade de início significativamente maior em relação à DP.

Unitermos: Doença de Parkinson, Doença de Parkinson Secundária, Epidemiologia, Diagnóstico.

Citação: Silva EG, Viana MA, Quagliato EMAB. Diagnóstico de síndromes parkinsonianas em uma clínica brasileira de distúrbios do movimento. Ver Neurociencias 2005; 13(4): 173-177.

\section{SUMMARY}

Introduction. Our study aimed to see the frequency of etiologies in the patients with parkinsonism (PK). Methods. We analyzed retrospectively 177 profiles of patients with parkinsonism attended in the Clinics Hospital of the State University of Campinas from January 1999 through December 2001, observing the anamnesis and the neuroimaging exams. Results. Of 177 profiles, 151 (85.3\%) had diagnostic criteria to Parkinson's disease (PK), 6 (3.4\%) to vascular PK (VP), 7 (4.0\%) to drug-induced PK (DIP), 6 (3.4\%) to Parkinson-plus (PP), 1(0.6\%), to pantetonatokinase deficiency, $1(0.6 \%)$ to spinocerebelar ataxia form 3, and 5 (2.8\%) unspecified PK (UP). Age onset of disease was $54 \pm 12$ years old to PK, $75 \pm 9$ to DIP, $60 \pm 14$ to VP and $53 \pm 9$ to PP. Conclusion. PK was the commonest form of parkinsonism in our clinic, followed by DIP, VP and PP. The patients with DIP are significantly older than patients with PK.

Keywords: Parkinson Disease, Parkinson Disease Secondary, Epidemiology, Diagnosis.

Citation: Silva EG, Viana MA, Quagliato EMAB. Diagnostic of parkinsonian syndrome in a Brazilian movement disorders clinic. Rev Neurociencias 2005; 13(4): 173-177.

Trabalho realizado: Faculdade de Ciências Médicas - Universidade Estadual de Campinas (FCM/UNICAMP)

1 - Graduando em medicina

2 - Médica do Departamento de Neurologia

3 - Professora adjunta do Departamento de Neurologia, responsável pelo Setor de Distúrbios do Movimento do Hospital das Clínicas da UNICAMP 


\section{INTRODUÇÃO}

As síndromes parkinsonianas (SP) dividem-se em três categorias principais: doença de Parkinson idiopática (DP); SP secundária a acidente vascular cerebral (AVC), infecções do sistema nervosa central, trauma craniencefálico, uso de neurolépticos ou outras drogas bloqueadoras de receptores dopaminérgicos ou que reduzem a dopamina, hidrocefalia, tumor cerebral; e SP ocorrendo em doenças neurodegenerativas - Parkinson-plus (PP) ${ }^{1,2}$. A DP é a forma mais comum de SP em diversos estudos $^{3}$, incluindo um estudo de população brasileira ${ }^{4}$, mas as causas secundárias variam de acordo com o local do estudo, portanto realizamos um levantamento das etiologias de SP em uma população brasileira de uma clínica de distúrbios do movimento, além de caracterizar clinicamente estes pacientes e pesquisar diferenças epidemiológicas entre os pacientes nos estudos.

\section{MATERIAIS E MÉTODOS}

Foi realizado um trabalho retrospectivo com os prontuários dos pacientes com os critérios diagnósticos das diferentes etiologias de SP. Solicitamos ao setor de informática do Hospital das Clínicas da Universidade Estadual de Campinas (HC/UNICAMP) a listagem de todos os pacientes com SP atendidos no Ambulatório de Distúrbios do Movimento do departamento de Neurologia do HC/UNICAMP de Janeiro de 1999 a dezembro de 2001. Foi-nos fornecido uma lista com 177 pacientes que apresentavam estas características. Os prontuários tinham toda a evolução clínica e resultado de exames dos pacientes.

Os pacientes moravam na região de Campinas (cidade localizada no estado de São Paulo a $95 \mathrm{Km}$ da capital do estado) com uma população de cerca de cinco milhões de habitantes, com predominância de brancos e nível socioeconômico baixo ou médio-baixo, e possui nosso hospital como referência terciária em saúde. Como é um hospital de referência terciária, todos os pacientes foram encaminhados para nosso Ambulatório para seguimento e investigação. Nosso estudo é retrospectivo, portanto os pacientes tinham diagnóstico, que foi feito a partir da evolução clínica da anamnese e exame neurológico, baseando-se nos critérios diagnósticos.

Para o diagnóstico de DP foram considerados critérios de inclusão: pelo menos dois dos seguintes sinais, tremor em atitude de repouso, acinesia/bradicinesia, rigidez e instabilidade dos reflexos posturais; início unilateral dos sintomas, desenvolvimento assimétrico da doença; resposta a agonista dopaminérgico boa ou excelente. Os critérios de exclusão para DP foram: demência, sinais piramidais ou cerebelares e disautonomia no início da doença (isto pode indicar PP) ou história de uso de substâncias tóxicas, ou encefalite e/ou relação entre um evento cerebrovascular e o início da SP ${ }^{1,2,5}$.
Os critérios diagnósticos para SP de causa vascular (PV) foram: evolução aguda ou subaguda; presença de rigidez ou acinesia; marcha com alargamento de base; fatores de risco vasculares presentes - hipertensão arterial sistêmica, AVC prévio, doença aterosclerótica sistêmica; neuroimagem mostrando pelo menos dois infartos nos gânglios da base ou doença difusa de substância branca; melhora espontânea do quadro clínico, sem usar levodopa ${ }^{6-8}$.

Os critérios para diagnóstico de SP induzida por drogas (PID) foram: presença de pelo menos dois dos sinais clínicos citados nos critérios de inclusão para DP; uma história de uso de agentes bloqueadores de receptor de dopamina por pelo menos 6 meses; ausência de sintomas precedendo o uso da droga?

Os critérios diagnósticos para PP foram: presença de bradicinesia ou rigidez, associada a outras anormalidades neurológicas, tais como alterações nas funções cognitivas, piramidais, cerebelares, autonômicas periféricas ou óculo-motoras e resposta deficiente ou nula à levodopa ${ }^{10}$.

Os pacientes que não apresentavam diagnóstico definitivo por falta de seguimento no ambulatório ou informação clínica insuficiente, foram classificados como SP de etiologia indeterminada (PI).

Os exames complementares de neuroimagem, tomografia computadorizada (TC - realizado em aparelho de tomografia computadorizada convencional SIEMENS Somaton $\mathrm{ART}$ ) e ressonância magnética ( $\mathrm{RM}$ - aparelho de ressonância nuclear magnética ELSCINT, com potência de dois Tesla), colaboraram para o diagnóstico diferencial, quando haviam dúvidas.

Os prontuários dos pacientes foram utilizados para coleta de dados de história clínica e exame neurológico, bem como o quadro evolutivo e os exames de imagem revisados, seguindo ficha no banco de dados do Programa EPI-INFO 6.04. Diferenças significativas foram consideradas quando $p<0,05$.

\section{RESULTADOS}

No Gráfico 1 é mostrada a freqüência das etiologias observadas nos prontuários analisados no período da pesquisa.

Os casos de PID estavam relacionados ao uso de cinarizina (três casos), flunarizina (dois casos) e neuroléptico (dois casos). Os pacientes com PP tinham como etiologia as seguintes afecções: atrofia de múltiplos sistemas (três casos), paralisia supranuclear progressiva (dois casos) e degeneração córtico-basal (um caso). As demais causas de parkinsonismo foram deficiência de pantetonato-quinase (síndrome de HallervordenSpatz) e ataxia espinocerebelar tipo 3 (AEC3 - doença de Machado-Joseph). 


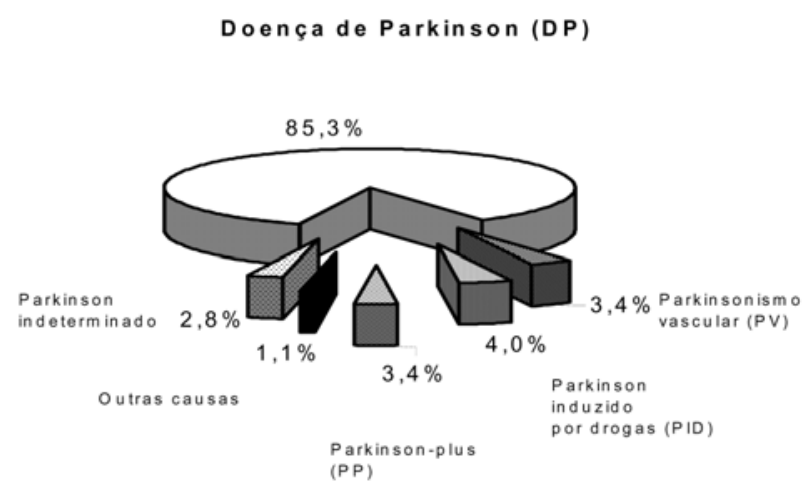

Gráfico 1. Freqüência de etiologias para os casos analisados $(n=177)$.

Havia um predomínio de pacientes do sexo masculino, totalizando 111 prontuários $(62,7 \%)$, sendo 96 com DP, 5 com PID, 4 com PV, 4 com PP e 2 com SP por outras causas; $93,8 \%$ (166) eram brancos e 26,6\% (47) apresentavam antecedente de tabagismo. Com relação à condição de moradia, 89,8\% (159) residiam em ambiente urbano, sendo que $47,1 \%$ (75) destes, apresentavam antecedente de vida rural; 93 pacientes $(52,5 \%)$ tiveram antecedente de utilização de água de poço. Apenas 1,1\% (2) dos pacientes apresentava antecedente familiar de tremor essencial e, 10,7\% (19) possuíam algum familiar com relação de primeiro grau com DP, sendo 16 com diagnóstico de DP.

$\mathrm{Na}$ Tabela 1, podem ser encontrados a média de idade de início de aparecimento dos sintomas iniciais e tempo de doença com seus respectivos desvios padrões, para cada classificação das principais etiologias de SP (média \pm desvio-padrão), sendo que a idade de início variou de 20 a 87 anos, com maior freqüência entre 40 e 69 anos (74,5\%).

$\mathrm{Na}$ Tabela 2, é observado o número de pacientes com idade de início da doença divididos em grupos etários com intervalo de 10 anos para cada uma das principais etiologias, separadas por sexo.
Tabela 1. Idade de início e tempo de doença.

\begin{tabular}{|ccc|}
\hline Diagnóstico & Idade de início (anos) & $\begin{array}{c}\text { Tempo de doença } \\
\text { (anos) }\end{array}$ \\
\hline DP & $54,33 \pm 12,36$ & $9,73 \pm 5,71$ \\
PV & $60,50 \pm 14,06$ & $9,66 \pm 3,93$ \\
PID & $75,85 \pm 9,38^{\star}$ & $6,57 \pm 2,57$ \\
PP & $53,00 \pm 9,87$ & $5,66 \pm 2,42$ \\
\hline
\end{tabular}

${ }^{*} p<0,05$ em relação à $D P$

DP: Doença de Parkinson; PV: Parkinsonismo vascular; PID: Parkinson induzido por drogas; PP: Parkinson-plus.

Foi analisada a presença de afecções associadas ao quadro parkinsoniano, ocorrendo em 74,0\% (131) dos pacientes, sendo as mais freqüentes, hipertensão arterial sistêmica (HAS) (36,7\% do total de prontuários) e depressão (10,7\%). Dos casos de HAS, 53 correspondiam a pacientes com DP, 4 a PID, 4 a PV, 1 a PP e 3 a PI; dos casos de depressão, 13 tinham DP, 3 com PV, 1 com PP e 2 com PI. Demência foi encontrada em $5,6 \%$ dos pacientes, sendo $1 \mathrm{com}$ DP, 2 com PID, 3 com PV e 4 com PP.

Dos casos de DP, 98 (64,9\%) apresentavam tremor de repouso no quadro clínico inicial, 45 (29,8\%) apresentavam bradicinesia, $32(21,2 \%)$ rigidez e $2(1,3 \%)$ instabilidade postural. Na Tabela 3, mostramos a freqüência de sinais clínicos no momento da análise dos prontuários na DP, PP e PV.

Com relação à lateralidade do aparecimento dos sinais, em $54,3 \%$ dos casos (82) o quadro iniciou-se à direita e em 45,7\% (69), à esquerda.

A TC e a RNM permitiram dar o diagnóstico etiológico em todos os casos de PV, além de servir como diagnóstico diferencial para casos de DP e outras SP. Dos pacientes com DP, 20 apresentavam doença vascular de substância branca observada ao exame de neuroimagem, de caráter inespecífico, não relacionado ao quadro clínico da doença.

Tabela 2. Idade e freqüência sexo-específica por etiologia de Síndrome Parkinsoniana.

\begin{tabular}{|c|c|c|c|c|c|c|c|c|c|c|c|c|}
\hline \multirow{2}{*}{ Idade de início (anos) } & \multicolumn{3}{|c|}{ DP } & \multicolumn{3}{|c|}{ PID } & \multicolumn{3}{|c|}{ PV } & \multicolumn{3}{|c|}{ PP } \\
\hline & $\mathbf{F}$ & M & total & $\mathbf{F}$ & M & total & $\mathbf{F}$ & M & total & $\mathbf{F}$ & M & total \\
\hline$<40$ & 5 & 15 & 20 & 0 & 0 & 0 & 0 & 1 & 1 & 0 & 1 & 1 \\
\hline $40-49$ & 10 & 20 & 30 & 0 & 0 & 0 & 0 & 0 & 0 & 0 & 1 & 1 \\
\hline $50-59$ & 14 & 28 & 42 & 0 & 1 & 1 & 1 & 0 & 1 & 2 & 1 & 3 \\
\hline $60-69$ & 20 & 25 & 45 & 0 & 0 & 0 & 1 & 2 & 3 & 0 & 1 & 1 \\
\hline $70-79$ & 5 & 7 & 12 & 1 & 3 & 4 & 0 & 1 & 1 & 0 & 0 & 0 \\
\hline$\geq 80$ & 1 & 1 & 2 & 1 & 1 & 2 & 0 & 0 & 0 & 0 & 0 & 0 \\
\hline
\end{tabular}

DP: Doença de Parkinson; PV: Parkinsonismo vascular; PID: Parkinson induz to por drogas; PP: Parkinson-plus. 
Tabela 3. Freqüência dos sinais clínicos no quadro atual nas Síndromes Parkinsonianas.

\begin{tabular}{|cccc|}
\hline Sinais clínicos & DP & PP & PV \\
\hline Tremor & $135(89,4 \%)$ & $3(42,9 \%)$ & $6(100 \%)$ \\
Bradicinesia & $129(85,4 \%)$ & $6(85,7 \%)$ & $6(100 \%)$ \\
Rigidez & $135(89,4 \%)$ & $4(57,1 \%)$ & $5(83,3 \%)$ \\
Instabilidade postural & $37(24,5 \%)$ & $3(42,9 \%)$ & $2(33,3 \%)$ \\
\hline
\end{tabular}

DP: Doença de Parkinson; PV: Parkinsonismo vascular;

PID: Parkinson induzido por drogas; PP: Parkinson-plus.

\section{DISCUSSÃO}

De forma similar a outros estudos $4,5,11-15$, a DP foi à forma de SP mais encontrada em nossa análise, compreendendo $85,3 \%$ de todos os casos cujos prontuários foram consultados neste hospital, porém os coeficientes apresentados devem ser vistos com cautela, já que o estudo foi realizado a partir do levantamento de prontuários de pacientes atendidos em um hospital de nível terciário, portanto os casos atendidos na unidade básica de saúde (centro de saúde) ou unidades de emergência (pronto-socorro) podem não ser encaminhados ao nosso serviço para acompanhamento, como nos casos de PID, já que muitos pacientes fazem uso dos medicamentos sem um controle muito rígido ${ }^{4}$, fazendo suspeitar que sua freqüência seja maior do que a encontrada.

PID, PV e PP foram as causas mais comuns de SP secundária, semelhante a outro estudo brasileiro ${ }^{4}$. Mas diferente deste, PID não foi uma etiologia muito freqüente, talvez porque estes casos não são encaminhados para nosso ambulatório, como comentado anteriormente.

DP foi encontrado com maior freqüência em homens, como observado na literatura ${ }^{6,16}$, em todas as faixas etárias, com exceção dos pacientes com mais de 80 anos.

Nossos resultados sugerem que viver em ambiente urbano ou rural, além de antecedente do uso de água de poço, não constitui fator suficiente para modificar o quadro de SP, como visto no estudo de Ferraz et $a /{ }^{17}$. Antecedente de tabagismo foi observado em número semelhante ao encontrado na população brasileira (aproximadamente 30\%), não representando qualquer relação de proteção contra a doença ${ }^{18}$.

Hipertensão e depressão foram as afecções mais freqüentemente associadas à $\mathrm{SP}$, sendo que hipertensão foi mais freqüente em nosso estudo do que no de Sutcliffe \& Meara $^{16}$, em que não ultrapassou $23 \%$, porém não constituindo fator de risco para o aparecimento das etiologias. Os casos de depressão e demência foram estatisticamente significativos ( $p<$ $0,05)$ ao se comparar as etiologias de DP e PV, havendo um maior risco para os pacientes do último grupo de apresentar estas doenças associada ao quadro extrapiramidal.

A idade de início dos sintomas concentrou-se entre 40 e 69 anos, porém havia número significativo de indivíduos com idade inferior a 40 anos (13,8\%) e, comparação com levantamento prévio em ambiente hospitalar ${ }^{19}$ indica que estão sendo observados pacientes mais jovens com DP, podendo representar um diagnóstico mais precoce da doença a partir do quadro clínico. Pode ser observado, ainda que os casos que apresentaram PID, tiveram o início da doença concentrada na faixa etária maior de 70 anos, apresentando idade mais elevada em relação aos pacientes com DP; os pacientes com PV apresentavam idade de início da doença concentrada a partir de 50 anos, porém sem relação estatisticamente significativa comparada às outras formas de SP.

Como esperado e observado em outros estu$\operatorname{dos}^{4,11,12,14,20}$, tremor, rigidez e bradicinesia foram as formas clínicas mais observadas na DP, porém instabilidade postural foi encontrada em número relativamente pequeno de indivíduos com DP, comparando-se com outros estudos ${ }^{4,11,14}$, talvez por ser o sinal clínico mais subjetivo para diagnóstico. Não houve relação significativa entre a lateralidade ao início da DP com a idade, fatores epidemiológicos, sinais clínicos ou afecções associadas, havendo apenas uma discreta predominância do lado direito comparado ao esquerdo (54\% vs $46 \%$ ), apresentando diferença em relação à pesquisa de Poewe \& Wenning ${ }^{20}$, que apresentou predomínio significativo do lado direito (61\% vs 39\%).

Os estudos de imagem dos pacientes mostram alterações de substância branca e pequenos infartos que nem sempre se correlacionam com a SP, podendo ser simples achados, portanto apesar de exames de neuroimagem normais serem sugestivos de DP, devese utilizar os critérios clínicos associados aos exames e evolução da doença para fazer o diagnóstico diferencial entre DP e PV.

\section{CONCLUSÃO}

Concluindo, DP é a forma de SP mais encontrada, sendo que as outras etiologias são pouco observadas em uma clínica de distúrbios do movimento de atendimento terciário.

\section{SUPORTE FINANCEIRO}

Fundação de Amparo à Pesquisa do Estado de São Paulo (FAPESP). 


\section{REFERÊNCIAS BIBLIOGRÁFICAS}

1. Larsen JP, Dupont E, Tandberg E. Clinical diagnosis of Parkinson's disease. Proposal of diagnostic subgroups classified at different levels of confidence. Acta Neurol Scand 1994;89:242-251.

2. Elbaz A, Bower JH, Maraganore DM, et al. Risk tables for Parkinsonism and Parkinson's disease. J Clin Epidemiol 2002:55:25-31.

3. Marsden CD. Parkinson's disease. J Neurol Neurosurg Psychiatry 1994;57:672-681.

4. Cardoso F, Camargos ST, Júnior GAS. Etiology of Parkinsonism in a Brazilian movement disorders clinic. Arq Neuropsiquiatr 1998;56(2):171-175.

5. Baldereschi M, Di Carlo A, Rocca WA, et al. Parkinson's disease and parkinsonism in a longitudinal study: Two-fold higher incidence in men. Neurology 2000;55:1358-1363.

6. Hurtig HI. Vascular parkinsonism, in Stern MB, Koller WC (eds). Parkinsonian Syndromes. New York: Marcel Dekker, 1993, 81-83.

7. Critchley M. Arteriosclerotic parkinsonism. Brain 1929;52:23-83.

8. Bennet DA, Wilson RS, Gilley DW, Fox JH. Clinical diagnosis of Binswanger'sdisease. J Neurol Neurosurg Psychiatry 1990;53:961-965.

9. Trenkwalder C, Schwars J, Gebhard J, et al. Stanberg trial on epidemiology of parkinsonism and hypertension in the elderly: Prevalence of Parkinson's disease and related disorders assessed by a door-to-door survey of inhabitants older than 65 years. Arch Neurol 1995;52:1017-1022.

10. Fahn S. Secondary parkinsonism. In Goldensohn and Appel (eds): Scientific Approaches to Clinical Neurology. Philadelphia: Lee \& Febiger, 1977, 1159.
11. Melcon $\mathrm{MO}$, Anderson DW, Vergara RH, Rocca WA. Prevalence of Parkinson's disease in Junín, Buenos Aires Province, Argentina. Movement Disorders 1997;12(2):197-205.

12. Hoehn MM, Yahr MD. Parkinsonism: onset, progression and mortality. Neurology 1967;17(5):S11-S26.

13. Gomes MM. Epidemiologia da doença de Parkinson. Arq Bras Med 1995;69(1):19-22.

14. Chen RC, Chang SF, Su CL, et al. Prevalence, incidence, and mortality of PD: A door-to-door survey in Ilan County, Taiwan. Neurology 2001;57:1679-1686.

15. de Rijk MC, Tzourio C, Breteler MMB, et al. Prevalence of parkinsonism and Parkinson's disease in Europe: the EUROPARKINSON collaborative study. J Neurol Neurosurg Psychiatr 1997;62:10-15.

16. Sutcliffe RLG, Meara JR. Parkinson's disease epidemiology in the Northampton District, England, 1992. Acta Neurol Scand 1995;92:443-450.

17. Ferraz, HB, Andrade LAF, Tumas V, Calia LC, Borges V. Rural or urban living and Parkinson's Disease. Arq Neuropsiquiatr 1996;54(1):37-41.

18. Sugita M, Izuno T, Tatemichi M, Otahara Y. Metaanalysis for epidemiologic studies on the relationship between smoking an Parkinson's disease. J Epidemiol 2001;11:87-94.

19. Teräväinen $H$, Forgach $L$, Hietanen $M$, Schulzer $M$, Schoenberg B, Calne DB. The age of onset of Parkinson's disease: etiological implications. J Canad Scienc Neurol 1986;13(4):317-319.

20. Poewe WH, Wenning GK. The natural history of Parkinson's disease. Ann Neurol 1998;44(Suppl 1):S1-S9. 